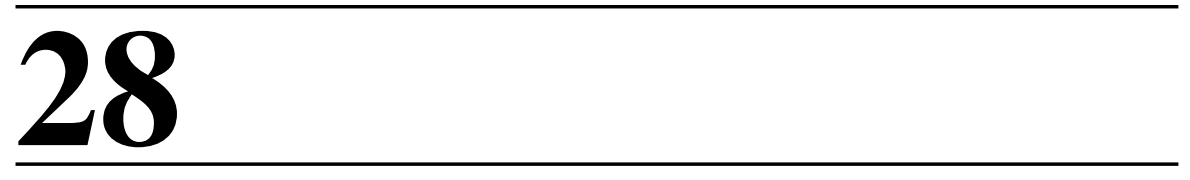

\title{
IN VITRO BIOLOGICAL TESTING IN THE DEVELOPMENT OF NEW DEVICES
}

\author{
Marta L. Alves Da Silva, Albino Martins, Ana \\ Costa-Pinto, Rui L. Reis, And Nuno M. Neves
}

3B's Research Group - University of Minho; Headquarters of the European Institute of Excellence on Tissue Engineering and Regenerative Medicine, AvePark, Parque de Ciência e Tecnologia, Zona Industrial da Gandra, 4805-017 Barco GMR - Portugal ICVS/3B's PT Government Associate Laboratory

\subsection{INTRODUCTION}

In general, scaffolds should be prepared with biocompatible materials, and if possible, already approved for clinical use. One of the crucial properties that can determine cell ingrowth, proliferation within the scaffolds and integration with the surrounding tissues is the 3D porous structure of the scaffolds [1]. It is also reported that an ideal scaffold for tissue engineering should be extremely porous and present a very interconnected pore network which allows nutrients and metabolic waste to flow [2]. The porous structure also contributes to provide cells the appropriate nutritional conditions and spatial organization for tissue regeneration [3], as well as providing a 3D environment for cell culture that is pivotal for their phenotype development and stability in vitro [4]. The scaffold should also degrade at the same time that of the tissue, without releasing toxic or inhibitory products. The mechanical properties of a scaffold should also be addressed, as they should match those of the native tissue being repaired, and the mechanical integrity should be maintained as long as needed for the integration of the newly formed tissue [5].

Biomaterials from Nature for Advanced Devices and Therapies, First Edition. Edited by Nuno M. Neves and Rui L. Reis. (C) 2016 John Wiley \& Sons, Inc. Published 2016 by John Wiley \& Sons, Inc. 
In vitro testing is performed before in vivo assays, as the newly developed materials can be examined outside of the body, the results are more reproducible, and more materials/conditions can be tested. The biocompatibility of any biomaterials should be evaluated in order to describe its strengths and feasibility for clinical manipulation [6]. So, preliminary in vitro tests are always carried out to screen and characterize the effects of a biomaterial or medical device prior its commercialization for humans [7].

\subsection{CYTOTOXICITY ASSAYS}

In vitro models for testing the biocompatibility of biomaterials and their derivatives are used to illustrate the hidden toxic effects of leachable materials or derivatives, such as catalysts, residual monomers or other polymer erosion products [6]. For example, the MEM extract test is designed to determine the rate of cytotoxicity of leachable materials released from medical materials or devices. In this method, cells are cultured with an extract prepared from the potential leachable material. After several time periods, the effect of the leachable materials on cells is assessed, evaluating parameters such as cell growth, metabolism, cell membrane damage, etc. The method is simple: a monolayer of cells is cultured with an extract of the medical material/device to be tested. The cells response along the time of reaction is then evaluated. If the extract contains toxic leachable materials originated from the medical material/device, cells will react. Therefore, cell growth inhibition, decrease or increase of metabolism, cell death, intracellular granulation and/ or change in cell morphology can be observed. The rate of cell damage is qualitatively and quantitatively established by a cytotoxicity index. When the value exceeds a certain level, the material or device fails the test. Cytotoxicity assays measure only effects on cells during the first 12 to 24 hours after exposure to toxic substances. However, many biological reactions in vivo are not simply cytotoxic and are propagated beyond 24 hours [6].

\subsection{EVALUATION OF CELL MORPHOLOGY AND DISTRIBUTION}

\subsubsection{Scanning Electron Microscopy (SEM)}

Scanning electron microscopy (SEM) is a microscopic method extremely valuable in polymeric materials and structures, along with many other research areas [8]. Its main attributes are that it reveals the 3D topography of the specimen examined and its magnification could be extremely high. SEM provides a direct assessment of pore size and structure. Interconnectivity of a scaffold can also be estimated, as well as examination of scaffold interior by sectioning the specimens. For polymeric structures, high magnifications are generally not possible because of the intensity of the electron beams damaging the thin polymeric samples, which generally leads to deformation and even melting of the specimen. To overcome this problem, polymeric samples are usually coated with conductive materials such as gold. 


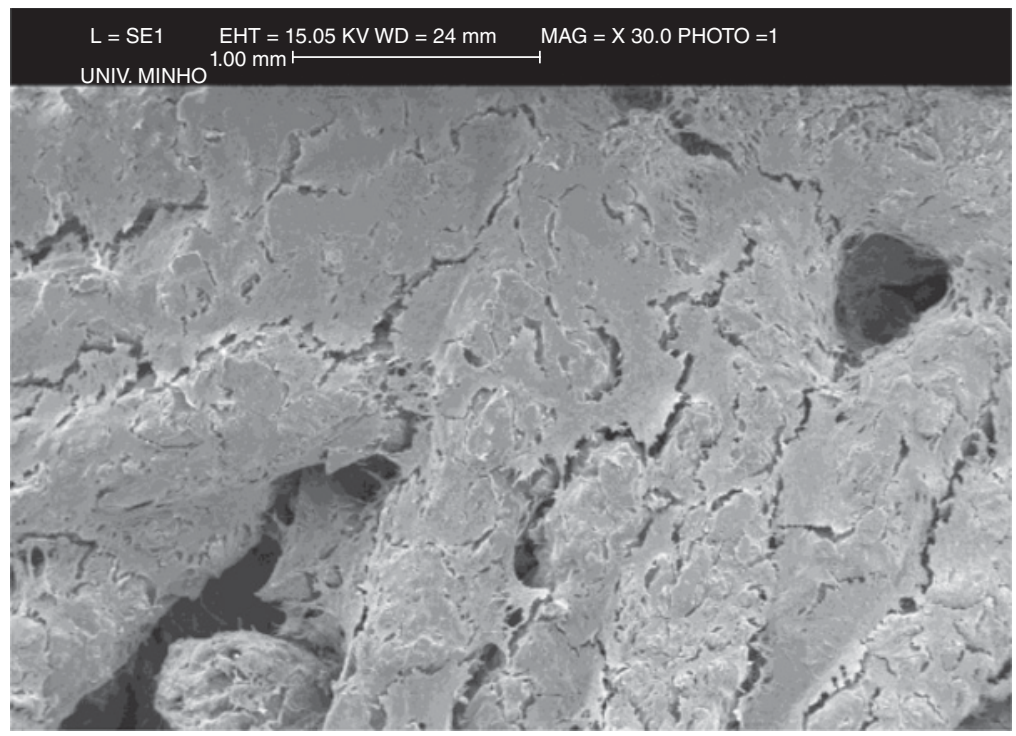

FIGURE 28.1 Human bone marrow mesenchymal stem cells cultured on chitosanpolybutylene succinate scaffolds for 7 days in osteogenic differentiated conditions.

\subsubsection{Fluorescence Microscopy}

In parallel to this indirect observation, the constructs can be analyzed by fluorescence microscopy. In this technique, the biological molecules are labeled with a fluorescent marker and detected visually, usually by selecting an appropriated wavelength [9]. One advantage of the fluorescence microscopy is the fact that some dyes quench the fluorescence of another dye by the so-called Förster energy transfer. Another advantage is that the environment influences the fluorescence wavelength; this can also be used to get more insight in the structural properties of a biomolecule. In other fluorescence-based microscopy, specifically in confocal microscopy, a laser beam is split and refocused just on the plane of interest [9]. This reduces one problem of normal fluorescence microscopy where the entire sample is illuminated and both in-focus and out-of-focus points contribute equally to the signal. The resolution reaches 200 $\mathrm{nm}$ in the $x y$-plane and $500 \mathrm{~nm}$ in the $z$-plane. The greatest advantage is the possibility of making 3D maps of the samples to within a depth of around 100-200 mm.

\subsubsection{Micro-Computed Tomography $(\mu \mathrm{CT})$}

Another technique used to analyze the tissue formation within the scaffolds is the micro-computed tomography (micro-CT). Microtomography (or micro-computed tomography, micro-computer tomography, X-ray tomographic microscopy, high resolution X-ray tomography) like computed tomography (CT), makes use of a X-ray source to create shadow cross-section images/projections of a $3 \mathrm{D}$ object that later can be used to recreate a virtual model without destroying the original object [10]. 
The term micro is used to indicate that the pixel sizes of the cross-sections are in the micrometer range. In general, there are two types of scanner setups. In one setup, the X-ray source and detector are typically stationary during the scan while the sample/animal rotates. The second setup, much more like a clinical CT scanner, is gantry based where the animal/specimen is stationary in space while the X-ray tube and detector rotate around. These micro-CT scanners are typically used for small animals imaging (e.g. bone, lung, cardiovascular, tumor and soft tissue), human samples (e.g. skin and bone biopsies), electronic pieces and microdevices, plastic foams and composite materials, wood and paper, microfossils and diamonds, and other studies for which minute detail is desired [11].

\subsection{CELL VIABILITY ASSAYS}

Viability and proliferation of cells is an important parameter to consider when using cell cultures. Viability assays are used to measure the proportion of viable cells after a potentially traumatic procedure, such as primary disaggregation, cell separation, or cryopreservation [12]. The net increase in the number of cells (i.e. the growth yield) is labor intensive and time consuming to set up and analyze, particularly when a large number of samples is involved, and the duration of each experiment may be anywhere from 2 to 4 weeks. Instead, a number of alternatives have been devised for assaying cells at higher densities, e.g. in microtitration plates. Indirect measurements of viability are mainly based on metabolic activity. Indeed, the increase in the number of cells is proportional to the increase in the total amount of protein or DNA, or continued metabolic activity. In these cases, cell survival is defined by the retention of metabolic ability by cell population, some time after removal of the toxic influence.

Cell viability can be determined by several tests, for example MTT, MTS or Alamar Blue assay. One of the most used assays is the MTT test, based on the tetrazolium salt MTT (3-(4,5-dimethylthiazol-2-yl)-2,5-diphenyl tetrazolium bromide), that measures only living cells [13]. In this assay, the yellow tetrazole MTT is reduced to purple formazan in living cells [13]. A solubilization solution is added to dissolve the insoluble purple formazan product into a colored solution. Then, the absorbance of this colored solution is quantified by measuring at a certain wavelength (usually between 500 and $600 \mathrm{~nm}$ ) by a spectrophotometer. The absorption maximum is dependent on the solvent employed [13]. Recent alternatives to the MTT testing have been recently developed. Water-soluble tetrazolium salts were established by introducing positive or negative charges and hydroxy groups to the phenyl ring of the tetrazolium salt, or better with sulfonate groups added directly or indirectly to the phenyl ring [14]. For example, WST-8 (2-(2-methoxy-4-nitrophenyl)-3(4-nitrophenyl)-5-(2,4-disulfophenyl)-2H-tetrazolium), has several advantages over MTT, namely being reduced outside the cells and yielding a water-soluble formazan. Moreover, WST assays can be read directly, giving a more accurate signal than MTT, and present decreased toxicity to cells [14]. Scaffolds made of chitosan and starch blends, with the ability to form a porous structure in situ, have been developed in our group. The biocompatibility of the scaffolds was evaluated using extracts and direct 
contact tests. Results with the MTT test showed that materials extracts were clearly non-toxic to L929 fibroblast cells [15].

Cell viability can be also determined by a colorimetric assay named CellTiter $96^{\circledR}$ AQueous One Solution Cell Proliferation Assay (Promega; USA). Briefly, this assay is based on the bioreduction of a tetrazolium compound [3-(4,5-dimethylthiazol-2-yl)-5-(3-carboxymethoxyphenyl)-2-(4-sulfofenyl)-2H-tetrazolium, inner salt (MTS)] into a brown formazan product that is soluble in culture medium [16]. This conversion is accomplished by the production of NADPH or NADH by the dehydrogenase enzymes existing in metabolically active cells. The quantity of formazan product is directly proportional to the number of living cells in culture, as measured by the amount of $490 \mathrm{~nm}$ absorbance in a microplate reader, after $3 \mathrm{~h}$ of incubation at $37^{\circ} \mathrm{C}$.

Another well-known viability test in mammalian cell cultures is based on the resazurin (7-Hydroxy-3H-phenoxazin-3-one 10-oxide) dye. It was introduced commercially initially under Alamar Blue trademark (Trek Diagnostic Systems, Inc), and now also available under other names such as AB assay, Vybrant (Molecular Probes) and UptiBlue (Interchim). Resazurin is a non-toxic, cell permeable compound that is blue in color and virtually non-fluorescent [17]. When it enters cells, it is irreversibly reduced to the pink colored and highly red fluorescent resorufin. Viable cells continuously convert resazurin to resorufin, increasing the overall fluorescence and color of the media surrounding cells. Resazurin based assays show excellent correlation when compared to reference viability assays and tritiated thymidine based techniques, while being much easier and safer to use for the user [18]. It also allows for longer studies (minimally toxic to living cells), works for adherents cells and bacteria/fungi and can be multiplexed with several chemiluminescent assays, such as cytokine assays, caspase assays to measure apoptosis, or reporter assays to measure a gene or a protein expression [18].

One of the first steps in a TE approach is to determine the scaffolds cytotoxicity. Chitosan-based scaffolds have been produced by melting and compression technologies and their cytotoxicity has been accessed. Standard tests (accordingly to ISO/EN 10993 part 5 guidelines), namely MTS test with a 24-h extraction period, have been performed, revealing that L929 cells had comparable metabolic activities to that obtained for the negative control [19]. Moreover, direct-contact assays were performed in these structures and it was shown that cells were able to colonize the scaffolds, showing high levels of viability [20].

\subsection{CELL PROLIFERATION ASSAYS}

Cell proliferation can be assessed indirectly by quantifying its metabolic activity. Measurements of DNA synthesis are often taken to be representative of the amount of cell proliferation [12]. Cell proliferation rates determination is often used to determine the response of cells to a particular stimulus, i.e. physical (e.g. topography of a substrate) or chemical (i.e. toxin or growth/differentiation factor). The most commonly used technique for measuring nucleic acid concentration is the determination 
of absorbance at $260 \mathrm{~nm}\left(\mathrm{~A}_{260}\right)$. The major disadvantages of the absorbance method are the large relative contribution of RNA and single-stranded DNA to the signal, the interference caused by contaminants commonly found in nucleic acid preparations, the inability to distinguish between DNA and RNA, and the relative insensitivity of the assay (an $\mathrm{A}_{260}$ of 0.1 corresponds to a $5 \mu \mathrm{g} / \mathrm{mL}$ double-stranded DNA solution). Alternatively, DNA content may be assayed by several fluorescence methods, including reaction with DAPI, PicoGreen or Hoechst 33258. The fluorescence emission of Hoechst 33258 at $458 \mathrm{~nm}$ is increased by interaction of the dye with DNA at $\mathrm{pH} 7.4$ and in high salt to dissociate the chromatin protein [21]. This method gives a sensitivity of $10 \mathrm{ng} / \mathrm{mL}$, but requires intact double-stranded DNA. For examples, The PicoGreen ${ }^{\circledR}$ dsDNA assay allows the detection of double stranded DNA, where the number of cells is directly correlated with the quantity of protein or DNA present in cells. Double-stranded DNA (dsDNA) is measured using an ultrasensitive fluorescent nucleic acid stain [22], along the culturing time.

The fluorescent bisbenzimide (Hoechst) dyes are part of a family of blue fluorescent dyes used to stain DNA [23]. Hoechst 33258 dye is relatively selective for dsDNA and in high salt does not show fluorescent enhancement in the presence of either protein or RNA. The dye binds specifically to the A-T base pairs in dsDNA resulting in an increase in fluorescence and a shift in the emission maximum from 500 to $460 \mathrm{~nm}$ [24]. Hoechst 33258 is quenched by Bromodeoxyuridine (BrdU), which is commonly used to detect dividing cells. Cells can integrate BrdU in newly synthesized DNA as a substitute for thymidine. When BrdU is integrated into DNA, it is supposed that the bromine deforms the minor groove so that Hoechst dyes cannot reach their optimal binding site [25]. Hoechst dyes are often used as substitutes for DAPI, because they are less toxic, which ensures a higher viability of stained cells. Hoechst tests can be combined with other viability assays for an exhaustive cytotoxic evaluation of a scaffold. For example, cryogelation technology was used to synthesize novel scaffolds from natural polymers like chitosan, agarose and gelatin and its evaluation was performed using Hoechst, Alamar Blue and MTT tests [26].

The development of implantable scaffolds has several requirements. As stated above, the toxicity evaluation is one of the main requirements that have to be positively filled in order to translate the technology for the clinics. Besides the cytotoxicity tests, the evaluation of other parameters such as the double stranded DNA and total protein are very important. Recently, the cytotoxicity, DNA and protein contents of ulvan extracted from green algae Ulva lactuca were quantified, resulting in the conclusion that ulvan can be considered as non-toxic in the range of concentrations studied [27].

\subsection{BIOCHEMICAL ANALYSIS}

\subsubsection{Glucose Consumption and Lactate Production}

Glucose $\left(\mathrm{C}_{6} \mathrm{H}_{12} \mathrm{O}_{6}\right)$ is a ubiquitous fuel molecule in biology [28]. It is oxidized through a series of enzyme-catalyzed reactions to form carbon dioxide and water, 
yielding the universal energy molecule ATP. Due to its importance in metabolism, glucose level is a significant parameter to be determined in cell culture [29], being also a key diagnostic parameter for many metabolic disorders. Increased glucose levels have been associated with diabetes mellitus, hyperactivity of thyroid, pituitary and adrenal glands. Decreased levels are found in insulin secreting tumors, myxedema, hypopituitarism and hypoadrenalism.

D-Glucose is phosphorylated to D-glucose-6-phosphate (G-6-P) in the presence of the enzyme hexokinase (HK) and adenosine-5'-triphosphate (ATP) with the simultaneous formation of adenosine-5'-diphosphate (ADP):

$$
\text { D-Glucose + ATP } \stackrel{H K}{\longrightarrow} \text { G-6-P + ADP }
$$

In the presence of the enzyme glucose-6-phosphate dehydrogenase (G6P-DH), G-6-P is oxidized by nicotinamide-adenine dinucleotide phosphate (NADP) to Dgluconate-6-phosphate with the formation of reduced nicotinamideadenine dinucleotide phosphate (NADPH):

$$
\text { G-6-P }+\mathrm{NADP}^{+} \stackrel{\text { G6P-DH }}{\longrightarrow} \text { D-gluconate-6-phosphate }+\mathrm{NADPH}+\mathrm{H}^{+}
$$

The amount of NADPH formed in this reaction is stoichiometric to the amount of D-glucose. Therefore, the increase in NADPH could be measured by means of its light absorbance at 334,340 or $365 \mathrm{~nm}$.

Lactate $\left(\mathrm{CH}_{3} \mathrm{CH}(\mathrm{OH}) \mathrm{COO}^{-}\right)$plays important roles in many biological processes. $\mathrm{L}(+)$-Lactate is the major lactate stereoisomer formed in human intermediary metabolism and is present in blood. $\mathrm{D}(-)$-Lactate is also present but only at about $1-5 \%$ of the concentration of $\mathrm{L}(+)$-Lactate. Abnormally high concentrations of lactate have been related to disease states such as diabetes and lactic acidosis, etc.

In the presence of D-lactate dehydrogenase (D-LDH), D-lactic acid (D-lactate) is oxidized to pyruvate by nicotinamide-adenine dinucleotide (NAD). The oxidation of L-lactic acid requires the presence of the enzyme L-lactate dehydrogenase (L-LDH):

$$
\begin{aligned}
& \text { D-Lactase }+N A D^{+} \stackrel{D-L D H}{\longleftrightarrow} \text { pyruvate }+N A D H+H^{+} \\
& \text {L-Lactate }+N A D^{+} \stackrel{L-L D H}{\longleftrightarrow} \text { pyruvate }+N A D H+H^{+}
\end{aligned}
$$

The equilibrium of these reactions lies on the side of lactate. By trapping pyruvate in a subsequent reaction catalyzed by the enzyme glutamate pyruvate transaminase (GPT) in the presence of L-glutamate, the equilibrium can be displaced in favor of pyruvate and NADH:

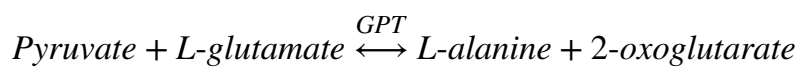

As for the glucose quantification, the amount of NADH formed in the above reactions of Lactate is stoichiometric to the amount of D-lactic acid and of L-lactic acid, 
respectively [30]. Consequently, the increase in NADH could also be determined by means of its light absorbance at 334,340 or $365 \mathrm{~nm}$.

\subsubsection{Protein Synthesis}

\subsubsection{Total Protein Quantification}

Total protein quantification is an important step for handling protein samples for isolation and characterization [31]. Total cellular protein varies in relation to cell growth, proliferation and differentiation [32-34]. Over the years, many different absorbancebased colorimetric methods have been developed to quantify protein, the most utilized of which rely on the reduction of copper in the presence of a chromogenic reagent $[35,36]$. Examples of total protein colorimetric methods are: the Lowry method, the bicinchoninic acid assay (BCA) and the biuret method [31]. While these methods work well, they are subject to interference by many compounds commonly used in protein purification, namely detergents and reducing agents. Alternatively, simple absorbance measurements of protein solutions at $280 \mathrm{~nm}$ (A280) can be performed, but are subject to interference from any nucleic acid contamination. In response to these difficulties, dye-binding protein assays were developed, being the most commonly used the method described by Bradford. This assay is subject to the formation of aggregates leading to a loss of signal over time. The most common procedures for the quantification of total protein are colorimetric methods, which are fast and simple.

The Lowry Assay is a common method for quantitation of soluble protein [35]. Due to its sensitivity, simplicity, and precision, it is often a method of choice. Based on two chemical reactions the first in which alkaline cupric tartrate complexes with the peptide bond of the protein and the second a reduction with Folin and Ciocalteu's phenol reagent. This reaction yields a purple color in which the absorption is read between 500 and $800 \mathrm{~nm}$. This assay may be performed directly with a protein solution, or a precipitation method involving deoxycholate and trichloroacetic acid may be used. Precipitation eliminates interference often caused by other reagents such as Tris, ammonium sulfate, EDTA, sucrose, citrate, and others. Protein concentration can then be determined using a calibration curve.

The principle of the bicinchoninic acid (BCA) assay is similar to the Lowry procedure, in that both rely on the formation of a $\mathrm{Cu}^{2+}$-protein complex under alkaline conditions, followed by reduction of the $\mathrm{Cu}^{2+}$ to $\mathrm{Cu}^{1+}$ [31]. The amount of reduction is proportional to the protein present. It has been shown that cysteine, cystine, tryptophan, tyrosine, and the peptide bond are able to reduce $\mathrm{Cu}^{2+}$ to $\mathrm{Cu}^{1+}$. BCA forms a purple-blue complex with $\mathrm{Cu}^{1+}$ in alkaline environments, thus providing a basis to monitor the reduction of alkaline $\mathrm{Cu}^{2+}$ by proteins. The BCA Assay is more sensitive and applicable than either biuret or Lowry procedures. In addition, it has less variability than the Bradford assay. The BCA assay has many advantages over other protein determination techniques: the color complex is stable; there is less susceptibility to detergents; it is applicable over a broad range of protein concentrations.

The Bradford Assay can be also used to determine the concentration of proteins in solution, consisting on the formation of a complex between the dye, Brilliant Blue G, 
and proteins. The protein-dye complex causes a shift in the absorption maximum of the dye from 465 to $595 \mathrm{~nm}$. The amount of absorption is proportional to the protein present. The Bradford assay requires no dilution and is suitable for micro, multiwell plate, and standard assays. The linear concentration range is $0.1-1.4 \mathrm{mg} / \mathrm{ml}$ of protein, using BSA (bovine serum albumin) as the standard protein. The Bradford assay is compatible with reducing agents, which are often used to stabilize proteins in solution. Other protein assay procedures (Lowry and BCA) are not compatible with reducing agents. The Bradford assay should be used in place of these protein assays if reducing agents are present. However, the Bradford assay is only compatible with low concentrations of detergents. If the protein sample to be assayed has detergents present in the buffer, it is suggested to use the BCA protein determination procedure.

\subsubsection{Enzyme-Linked Immunosorbent Assay}

The enzyme-linked immunosorbent assay (ELISA) is a commonly used technique for the determination of known analytes (e.g. proteins, peptides, hormones, etc.) [37]. ELISAs are routinely used in scientific research, veterinary medicine, environmental and agricultural applications, and in healthcare [38]. The fundamental principle of the ELISA is that the target analyte (the antigen) is recognized with high specificity by antibodies, which are proteins produced by the immune system. These antibodies can recognize and bind to the antigens, the labeling of the bound antibody forms the basis of the detection.

The success of an ELISA assay is dependent upon the underlying level of immunoreactivity of the capture and detection antibodies to the target analyte. Figure 28.2 shows one format of the assay, which uses two separate antibodies: the first to recognize and bind the target analyte, the second to detect the bound target. In a first step, one of the antibodies is applied to the well of a microtitre plate: this is known as the capture antibody. The capture antibody binds to the plate via passive adsorption and this step is often performed at $4^{\circ} \mathrm{C}$ overnight. A blocking solution, typically milk protein (casein), bovine serum albumin or fish gelatin, is applied. These proteins adhere to any vacant sites on the plastic surface of the well that are not occupied by capture antibodies thereby minimizing the effect of nonspecific binding by other

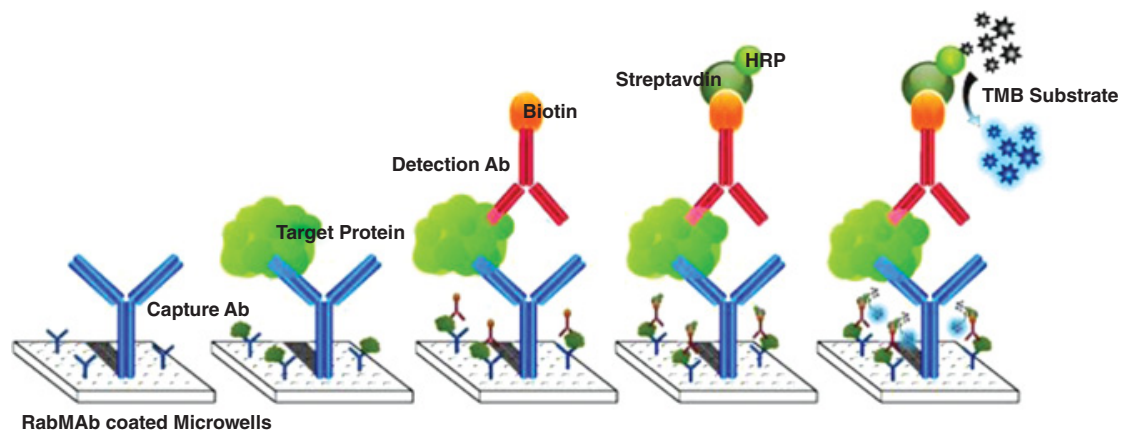

FIGURE 28.2 Scheme of a typical ELISA. For a color version of this figure, see the color plate section. 
reagents to the plate surface during subsequent incubation steps. Excess blocking agent is removed and the plate is rinsed before addition of the test sample (wash steps are incorporated between all incubation steps to minimize the background signal due to nonspecific binding).

If the test sample contains the target analyte, this will be bound by the capture antibody that is anchored to the plate (Step 2). After the incubation step with the test sample, the plate is washed prior to the addition of the enzyme-linked detection antibody (Step 3). The detection antibody is conjugated with an enzyme, which is commonly either horseradish peroxidase (HRP), alkaline phosphatase (AP) or $\beta$-D-galactosidase. These enzymes are proteins that catalyze the hydrolysis of a chromogenic substrate, such as 3,3',5,5'-tetramethylbenzidine or 2,6- dichlorophenolindophenol, which undergoes a colorimetric change that is measurable using a spectrophotometric plate reader at specified wavelengths (Step 4). With the catalysis of the traditional chromogenic substrates the reaction is terminated by the addition of a stop solution prior to measuring the absorbance of each of the wells of the microtitre plate.

In addition to the direct detection method, as illustrated in Figure 28.2, ELISAs may utilize an indirect detection approach. In this instance, as illustrated in Step 5, the secondary antibody used will recognize and bind to the appropriate species-specific sub-class of the antibodies (or immunoglobulins). It is the secondary detection antibody that is conjugated to the enzyme, as opposed to the primary antibody. This method offers versatility because a single enzyme labeled secondary detection antibody may be used for all assays involving the use of single host species of primary antibodies. The sensitivity of the ELISA may also be enhanced as the secondary antibody tends to be polyclonal, and this affords signal amplification via the recognition of multiple epitopes on the primary antibody.

\subsection{GENOTYPIC EXPRESSION ANALYSIS}

The determination of levels of gene expression in tissue engineered constructs is normally performed by several techniques that use extracted messenger ribonucleic acid (RNA). Natural-based scaffolds present a challenge when considering attaining high quality RNA for an efficient biological evaluation, as these materials interfere with the most usually used RNA extraction techniques [39]. The majority of the commercial RNA isolation kits are based on the use of an acid guanidinium thiocyanate-phenolchloroform extraction reagent, as first described by Chomczynki and Sacchi [40]. The TRIzol ${ }^{\circledR}$ family of reagents (Invitrogen, Carlsbad CA) is based on this method. This reagent is ready-to-use for isolation of total RNA from cells and tissues. Trizol disrupts cells and dissolves their components, while maintaining RNA integrity. Then, chloroform is added, which allows the separation of the solution into two distinct phases: aqueous and organic. RNA will remain in the aqueous phase and it is recovered by precipitation with isopropyl alcohol. When using natural polymers, the large polysaccharide fragments present after the digestion can entrap nucleic acids, while the small polysaccharides fragments can co-precipitate with the RNA in the 
precipitation step [39], leading to low RNA yields or interferences with the next analysis steps. Due to the high content in polysaccharides present in natural polymers, these RNA extraction TRIzol-based methods and commercial kits have shown several constrains in the isolation of quality RNA. Therefore, some researchers have been developing cetyltrimethylammonium bromide (CTAB)-ammonium acetate protocols for total RNA isolation from for example cotton [41] or from 3D chitosan, agarose and collagen matrices [39].

Real-time PCR (RT-PCR) is a quantitative technique based in the Polymerase Chain Reaction (PCR) procedure. PCR is a widely used technique that allows the amplification of a copy of a DNA fragment millions of times amplifying its number to detectable quantities (amplicon). This technique relies on thermal cycling and enzyme (DNA polymerase) replication of DNA. One major drawback of PCR is that the amplicon can only be visualized when the reaction finishes. In RT-PCR this does not happen, since this technique allows the amplification and simultaneous quantification of the amplicon. RT-PCR is usually combined with reverse transcription to quantify messenger RNA in cells or tissues. The difficulties in the RNA isolation that were referred previously can affect the RT-PCR reaction because of the low quality of the isolated RNA. Nevertheless, researchers have managed to overcome these difficulties and to perform this important technique in TE constructs for cartilage $[42,43]$, bone [44], vascularization [45] and several other applications.

\subsection{HISTOLOGICAL ASSESSMENT}

Prior to sectioning, samples need to be processed. In the case of cells or tissues, several embedding media are available for covering the structure and preserving its integrity. Depending on the sample its characteristics, various embedding media can be selected, such as paraffin, OCT compound or glycol methacrylate. The main difference between these media is on the processing technique. Paraffin samples are processed using high temperatures (between $60-80^{\circ} \mathrm{C}$ ) whereas OCT samples are processed in cold conditions using liquid nitrogen. Glycol methacrylate embedded samples are processed using intermediate temperatures $\left(4^{\circ} \mathrm{C}\right)$. Some natural polymers cannot stand the high temperatures used for paraffin processing, so the glycol methacrylate technique is used, for example for processing chitosan-poly(butylene terephthalate adipate) (CPBTA) scaffolds [46].

\subsubsection{Hematoxylin-Eosin}

Hematoxylin-eosin (H\&E) staining is a method widely used for the analysis of cells distribution and morphology. The combination of two stains (hematoxylin and eosin) enables the detection of cell nuclei and cytoplasm. This stain can be performed to evaluate the cells' distribution within scaffolds. The next figure shows two examples of H\&E stain of cells seeded onto natural-based scaffolds: compression molded chitosan-PBS scaffolds (Fig. 28.3a) and starch-polycaprolactone (SPCL) electrospun nanofiber meshes (Fig. 28.3b), seeded with bovine articular chondrocytes. 


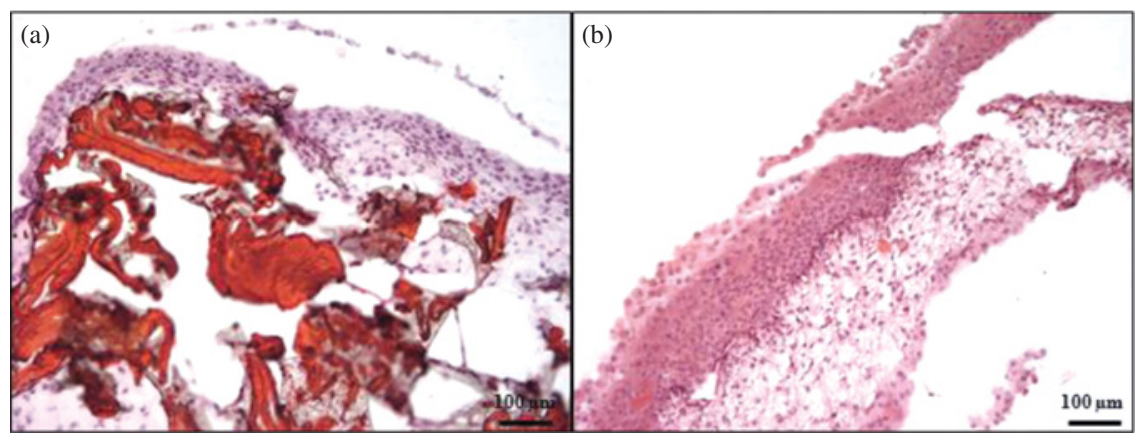

FIGURE 28.3 Bovine articular chondrocytes seeded onto natural-based scaffolds. (a) Chitosan-PBS scaffold; (b) SPCL electrospun nanofiber mesh. For a color version of this figure, see the color plate section.

\subsubsection{Immunodetection of Specific Proteins}

Immunohistochemistry is a technique performed to detect and identify specific protein markers on in vitro cultures cells and in tissues. Particularly important in defining the phenotype of the differentiating stem cells is an understanding of the tissue development in relation to gene expression of the cells [47]. This technique provides in situ information of the localization of a specific protein, and has been developed from the antigen-antibody binding reaction [48]. Immunohistochemistry methods can be classified as immunofluorescence, immunoenzymological staining and affinity histochemistry, depending on the different biotins conjugated with antibodies. The immunofluorescence method relies on the antigen-antibody binding reaction, where antigens are visualized by fluorescence dyes conjugated with antibodies, when activated by exciting light of specific wavelength under fluorescence microscope. The confocal microscope is often used to visualize samples stained with this technique. Figure 28.4 shows human articular chondrocytes seeded onto a polycaprolactone (PCL) membrane, which surface has been modified with chondroitin sulphate, a natural component of cartilage ECM. The fluorescent dies enable to visualize the cells morphology and arrangement in the scaffold.

In the immunoenzymological staining, enzyme-labeled antibodies are used to bind specific antigens. Afterwards, an enzyme substrate is added in order to generate insoluble particles that can be localized under light microscope or electronic microscope. This technique is considered to be more accurate in the localization of protein, as well as presenting a better contrast ratio. Figure 28.5 shows the result of the immunolocalization of collagen type II in a TE construct where bovine articular chondrocytes were seeded and cultured under dynamic conditions using a rotator inside the incubator.

\subsection{IN VITRO ENGINEERED TISSUES}

\subsubsection{Bone}

Bone tissue engineering may constitute the needed breakthrough technology to solve the problem of bone shortage in various destructive clinical conditions and 


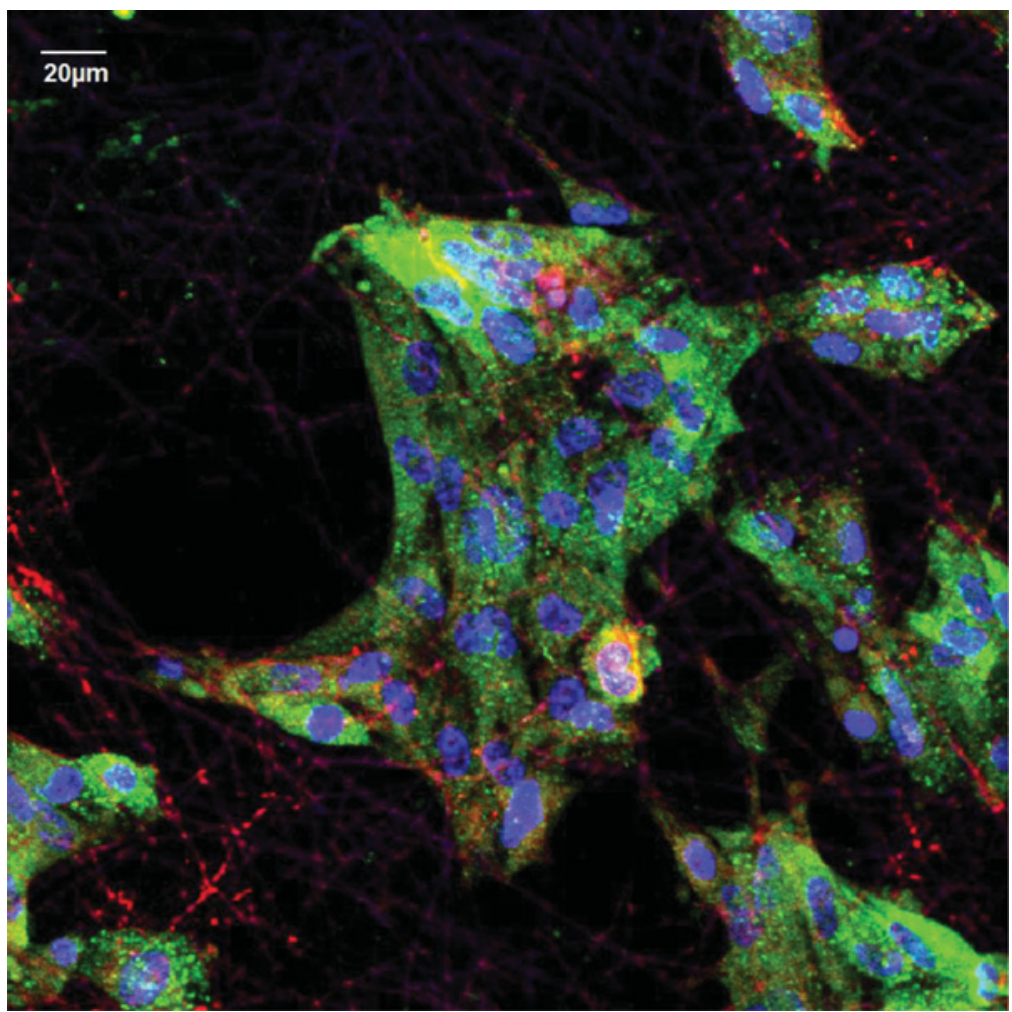

FIGURE 28.4 Confocal microscopy images of hACs seeded onto CS-immobilized PCL nanofiber meshes, at 21 days of culture. Cytoskeleton evidenced by phalloidin is shown in red, nuclei are stained blue for DAPI and vinculin is stained green. For a color version of this figure, see the color plate section.

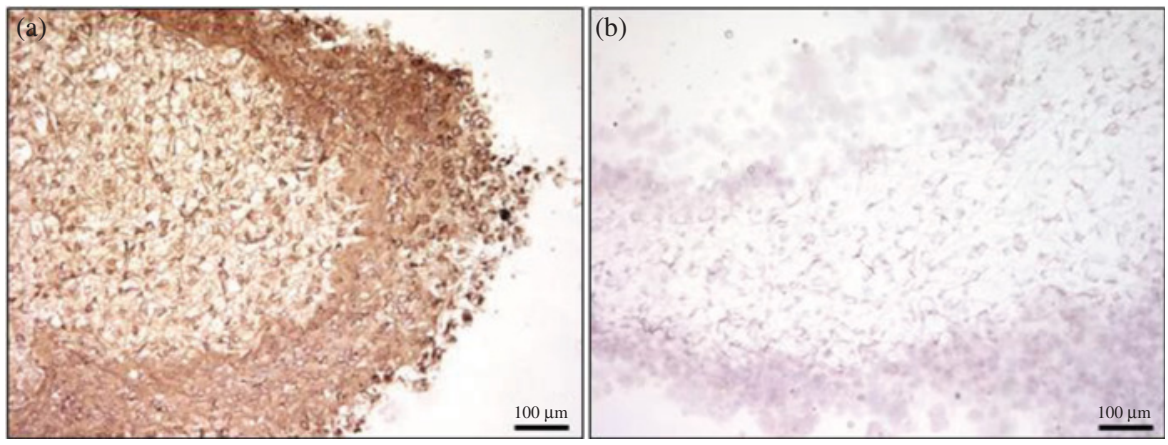

FIGURE 28.5 Optical microscopy images presenting the results obtained from the immunolocalization of collagen type II in histology sections of bovine articular chondrocytes seeded onto starch-compounded PCL nanofiber meshes cultured in dynamic conditions. Images present results at 4 weeks of culture for normal goat serum - control (B), and collagen type II (A). For a color version of this figure, see the color plate section. 
deformities, by providing functional tissue engineered biological substitute [49]. The most used approach in the field is based on seeding and in vitro culture of autologous primary cells (osteoblasts or adult stem cells differentiated into the osteogenic phenotype) on 3D scaffolds. These constructs will be further implanted into the bone defect. The scaffold will provide the adequate 3D environment for cells to adhere, proliferate and differentiate, while cells will synthesize ECM, producing bone tissue.

Culture of osteogenic cells depends on the adequate supplementation of their growth medium with a source of inorganic phosphate [50] - $\beta$-glycerophosphate, a nonphysiological organic substrate of alkaline phosphatase (ALP) [51], in order to produce mineralized ECM. Ascorbic acid is essential for the survival of human osteoblasts in vitro [52]. This osteogenic inducing agent is required for collagen synthesis and alkaline phosphatase activity [53]. Dexamethasone is a glucocorticoid that increases the expression of several genes associated with osteogenic differentiation [54]. Osteogenic differentiation process involves: (i) cell proliferation; (ii) ECM deposition and maturation; and (iii) mineralization [55].

\subsubsection{ALP Quantification}

A thorough analysis of mineralization and the progression of differentiation rely on biochemical assays. One of the most common used is the determination of the enzymatic activity of early marker ALP. High ALP activities are associated with active formation of mineralized matrix, and highest levels are found in the mineralization front in bone healing. Briefly, the activity of ALP was assessed using the $p$-nitrophenol assay. Nitrophenyl phosphate disodium salt is colorless, and is hydrolyzed by alkaline phosphatase, produced by cells, to form free $p$-nitrophenol, which is yellow. The reaction is stopped by the addition of $2 \mathrm{M} \mathrm{NaOH}$ and the absorbance read at $405 \mathrm{~nm}$. Standards are prepared with $p$-nytrophenol solution, to obtain a standard curve. ALP concentration is read off directly from the standard curve.

\subsubsection{Mineralization Assessment}

Total calcium content can also be quantified, by colorimetric or fluorimetric kits. With the onset of mineralization, ECM protein genes become up-regulated, like osteopontin and osteocalcin that are increasingly expressed with the accumulation of mineral [55]. The mineral content of the bone ECM can be qualitatively assessed by alizarin red or von Kossa staining and the calcium content can be quantitatively assessed. This information can be complemented by the analysis of the mineral fraction by energy dispersive spectroscopy to detect the presence of calcium and phosphorous elements, thin-film X-ray diffraction to analyze the crystallinity of the ECM and Fourier-transformed spectroscopy to detect the carbonate and phosphate groups [56,57].

Energy dispersive spectroscopy (EDS) methodology can be used to detect the presence of calcium $(\mathrm{Ca})$ and phosphorous $(\mathrm{P})$ elements by analyzing the surface 
of the constructs. These two chemical elements are constituents of the mineral phase (hydroxyapatite) of bone ECM. Their presence positively indicates the formation of mineralized ECM at the surface of the constructs. Fourier transform infrared spectroscopy (FTIR) method is used to study the chemical structure of a polymer, but it can also be used to analyze the chemical composition of the surface of a scaffold. In bone tissue engineering, it can be used to confirm the results obtained from EDS analyses, indicating the presence of phosphate and carbonate groups, which are typical for carbonated apatite.

\subsubsection{Specific Histological Stains}

Von Kossa and Alizarin red are the most frequently used stains to detect the mineralization process in osteoblast culture. Both stains are used to detect the mineralization, but the reactions that generate a positive staining are different. In fact the Von Kossa staining is based on a reaction of the silver nitrate that allows visualization of phosphate and carbonate anions that stain with a black color, while alizarin red $\mathrm{S}$ reacts with calcium and other cations staining red [58] (Fig. 28.6). Von Kossa staining can also react with other types of phosphate or carbonate salts, such as sodium phosphate staining black and generating false positives. Therefore, the Von Kossa stain is not specific for calcium, while alizarin red $\mathrm{S}$ reacts specifically with calcium and can therefore be considered a more reliable stain to detect mineralization.

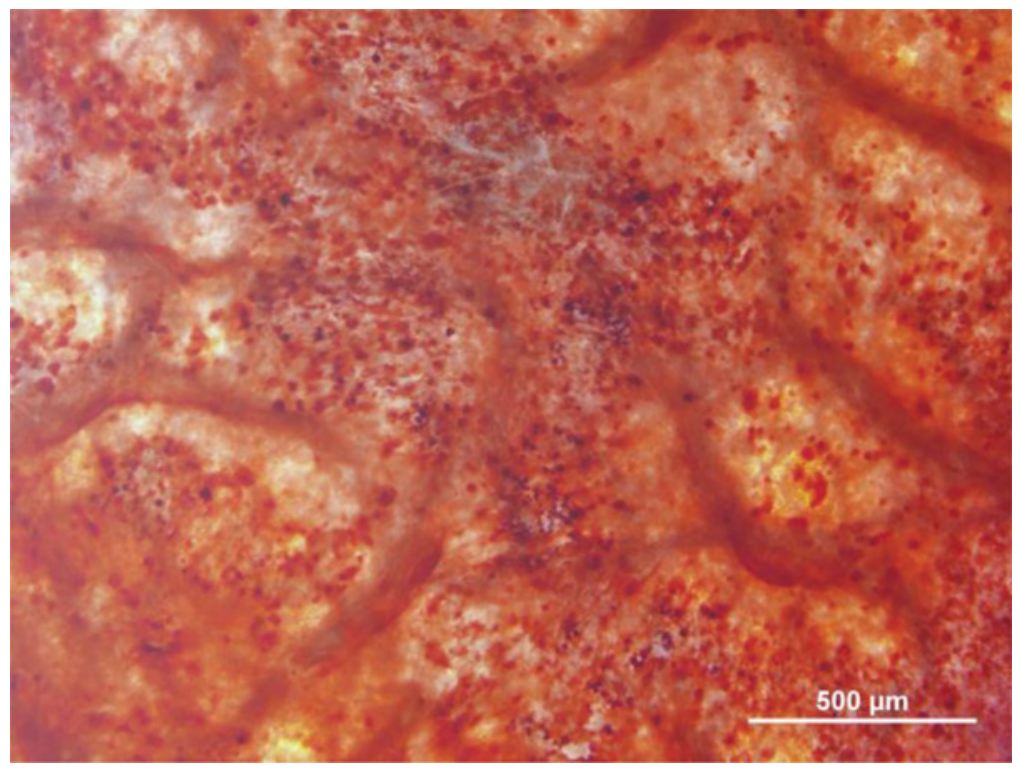

FIGURE 28.6 Alizarin red staining on mesenchymal stem cells differentiating to the osteogenic lineage, at 14 days of culture, seeded onto nanofiber meshes. For a color version of this figure, see the color plate section. 


\subsubsection{Cartilage}

\subsubsection{Glycosaminoglycan Quantification}

Adult articular cartilage is a hypocellular tissue, with a viscoelastic extracellular matrix (ECM) composed mainly by water, collagens, glycosaminoglycan (GAG) bearing proteoglycans (aggrecan, biglycan, deocrin and fibromodulin), hyaluronic acid and noncollagenous proteins (COMP, link). Quantitation of these components can be performed by their solubilization through exhaustive proteolitic digestion. Papain will fully solubilize GAG, by cleaving the core protein, and chondrocyte DNA, by degrading nuclear binding proteins. Digestion at high temperatures $\left(60^{\circ} \mathrm{C}\right)$ melts the collagen helix, thereby facilitating collagen proteolysis. The GAG content can be measured by using a colorimetric assay such as the dimethyl methylene blue (DMB) assay.

Glycosaminoglycan (GAG) quantification was based on the use of proteolytic enzymes able to degrade cartilage ECM and a colorimetric assay to measure the quantity of GAGs released [59]. Enzymes will degrade proteoglycans and the released GAGs will be quantified indirectly. The basic die 1,9 dimethylmethylene blue will link to the negatively charged GAGs and allow their detection by measuring the solution absorbance. The quality of engineered cartilage and the amount of GAGs deposited by bovine articular chondrocytes when seeded onto chitosan-based scaffolds have been quantified by this method [60].

\subsubsection{Collagen Content Determination}

Hydroxyproline is one of the main components of the collagen protein. The determination of the total collagen contents of a cartilage TE construct can be indirectly made by measuring the hydroxyproline amount. The spectrophotometric-based hydroxyproline assay is one of the few assays for collagen content and concentration quantification in several organs, including cartilage. This assay was described by Neuman and Logan (1950), and relies in the principle that all collagens contain globular domains and share the common structural motif of triple helical segments [61]. Collagen is one of the few proteins containing the amino acid hydroxyproline. Based on the absolute quantitation of hydroxyproline, which represents a fixed percentage of the amino acid composition of collagen in most mammalian tissues [62], the collagen content may be derived. Since its description [61], this method has been altered by several researchers in order to overcome some difficulties including its reproducibility between the same replicates, interferences by other components or differences in standard curves [63]. One of the most frequently used methods that has been adapted from the original is described by Bergman and Loxley [64]. Briefly, the collagen is converted to a soluble form (gelatin), by hydrolysis under high temperatures, and thus extracted from elastin and other substances that are not dissolved by the process. The collagen content is then estimated from the hydroxyproline content of acid hydrolysates of the extract [65]. 


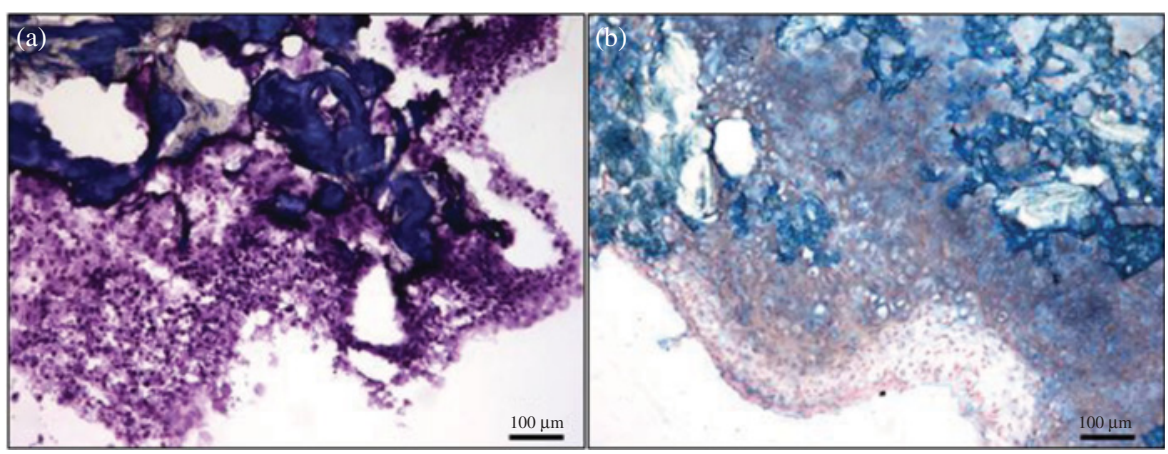

FIGURE 28.7 Examples of toluidine (a) and alcian blue (b) staining evidencing the cartilage ECM deposited by bovine articular chondrocytes onto chitosan-based scaffolds, at 28 days of culture. For a color version of this figure, see the color plate section.

\subsubsection{Specific Histological Stain}

Toluidine blue staining allows a metachromatic staining of cartilage matrix and specifically of its proteoglycan content. It stains proteoglycans and GAGs (Fig. 28.7a). Alcian blue stain is used to detect cartilage formation. This dye stains acid mucopolysacharides and GAGs (Fig. 28.7b). Safranin O staining is another technique to detect cartilage components. This is a cationic dye that in dehydrated cartilage sections gets its orthochromatic form, and is in this form that safranin $\mathrm{O}$ links to tissues GAGs.

\subsection{CONCLUDING REMARKS}

In vitro testing of biomaterials is essential for evaluation of cellular response to the scaffolds or devices and is the first step towards its final application in vivo. Cytotoxicity tests comprise cells morphological analyses, response to extracts and direct contact of cells and scaffolds. Evaluation of cell behavior when seeded onto the scaffolds can be made by various techniques such as SEM, confocal microscopy, proliferation assays, biochemical evaluation, histological stain or gene analysis. When working with natural materials, researchers must be able to adapt some of the referred techniques to the specificity of the biomaterials, as their high polysaccharide content may influence the outcome of the analysis. By the end of a positive outcome, the natural scaffold is then accepted to continue its evaluation in vivo.

\section{REFERENCES}

[1] Bagherzadeh, R. et al., Journal of Biomedical Materials Research Part A 2014, 102A, 903.

[2] Blakeney, B. A. et al., Biomaterials 2011, 32, 1583. 
[3] Pham, Q. P. et al., Biomacromolecules 2006, 7, 2796.

[4] Wang, Y. et al., Biomaterials 2005, 26, 7082.

[5] Freshney, R. I. In Culture of Cells for Tissue Engineering; Vunjak-Novakovic, G., Freshney, R. I., Eds.; John Wiley Sons, Inc.: New Jersey, 2006, p 3.

[6] Keong, L. C.; Halim, A. S. Int J Mol Sci 2009, 10, 1300.

[7] Marques, A. P. et al., Biomaterials 2002, 23, 1471.

[8] Hasirci, V.; Hasirci, N. In Surface and interfaces for biomaterials; Vadgama, P., Ed.; Woodhead Publishing Limited and CRC Press LLC: Cambridge and Boca Raton, 2005, p 29.

[9] Ziegler, C. In Surface and interfaces for biomaterials; Vadgama, P., Ed.; Woodhead Publishing Limited and CRC Press LLC: Cambridge and Boca Raton, 2005, p 200.

[10] Ho, S. T.; Hutmacher, D. W. Biomaterials 2006, 27, 1362.

[11] Ritman, E. L. In Annual Review of Biomedical Engineering 2004; Vol. 6, p 185.

[12] Freshney, R. I. In Culture of Animal Cells: A Manual of Basic Technique; Fifth ed.; Freshney, R. I., Ed.; John Wiley \& Sons, Inc.: 2005, p 359.

[13] Mosmann, T. J Immunol Methods 1983, 65, 55.

[14] Bernas, T.; Dobrucki, J. Cytometry 2002, 47, 236.

[15] Martins, A. M. et al., Acta Biomater 2008, 4, 1637.

[16] Cory, A. H. et al., Cancer communications 1991, 3, 207.

[17] Bueno, C. et al., Photochem Photobiol 2002, 76, 385.

[18] UptiBlue Viable Cell Counting Kit

[19] Correlo, V. M. et al., J Biomed Mater Res A 2009, 91, 489.

[20] Correlo, V. M. et al., Macromol Biosci 2010, 10, 1495.

[21] Labarca, C.; Paigen, K. Analytical biochemistry 1980, 102, 344.

[22] Chadwick, R. B. et al., BioTechniques 1996, 20, 676.

[23] Latt, S. A. et al., Journal of Histochemistry \& Cytochemistry 1975, 23, 493.

[24] Daxhelet, G. A. et al., Anal Biochem 1989, 179, 401.

[25] Kubbies, M.; Rabinovitch, P. S. Cytometry 1983, 3, 276.

[26] Bhat, S.; Kumar, A. Journal of Bioscience and Bioengineering 2012, 114, 663.

[27] Alves, A. et al., Phytother Res 2013, 27, 1143.

[28] Tamaru, S. et al., Org Biomol Chem 2014, 12, 815.

[29] Chiarini, A. et al., Biomaterials 2003, 24, 789.

[30] Chiu, L. L. Y.; Radisic, M. Biomaterials 2010, 31, 226.

[31] Krohn, R. I. In Current Protocols in Cell Biology; John Wiley \& Sons, Inc.: 2001.

[32] Engelhard, H. H. et al., Cytometry 1991, 12, 68.

[33] Marti, M. et al., Nat. Protoc. 2013, 8, 223.

[34] Kavya, K. C. et al., J Biomed Nanotechnol 2012, 8, 149.

[35] Lowry, O. H. et al., J Biol Chem 1951, 193, 265.

[36] Smith, P. K. et al., Anal Biochem 1985, 150, 76.

[37] Gan, S. D.; Patel, K. R. J Invest Dermatol 2013, 133, E10.

[38] Wang, X. Q. et al., J Control Release 2009, 134, 81.

[39] Wang, L. M.; Stegemann, J. P. Biomaterials 2010, 31, 1612. 
[40] Chomczynski, P.; Sacchi, N. Anal Biochem 1987, 162, 156.

[41] Zhao, L. et al., Phytochem Analysis 2012, 23, 647.

[42] Alves da Silva, M. L. et al., J Tissue Eng Regen Med. 2013, 2013 Oct 24. doi: 10.1002/term.1812. [Epub ahead of print].

[43] Alves da Silva, M. L. et al., Biomacromolecules 2010, 11, 3228.

[44] Costa-Pinto, A. R. et al., Biomacromolecules 2009, 10, 2067.

[45] Santos, M. I. et al., Biomaterials 2009, 30, 4407.

[46] Alves da Silva, M. L. et al., J Tissue Eng Regen Med 2011, 5, 722.

[47] Hofmann, S. et al., In Culture of Cells for Tissue Engineering; Vunjak-Novakovic, G., Freshney, R. I., Eds.; John Wiley \& Sons, Inc.: New Jersey, 2006, p 323.

[48] Houghten, R. A. P Natl Acad Sci USA 1985, 82, 5131.

[49] Langer, R.; Vacanti, J. Science 1993, 260, 920.

[50] Bellows, C. G. et al., Bone and Mineral 1992, 17, 15.

[51] Gerstenfeld, L. C. et al., Developmental Biology 1987, 122, 49.

[52] Koshihara, Y. et al., Biochemical and Biophysical Research Communications 1987, 145, 651.

[53] Takamizawa, S. et al., Cell Biology International 2004, 28, 255.

[54] Subramaniam, M. et al., Journal of Cellular Biochemistry 1992, 50, 411.

[55] Lian, J. B.; Stein, G. S. Critical Reviews in Oral Biology \& Medicine 1992, 3, 269.

[56] Weiner, S. Journal of Structural Biology 2008, 163, 229.

[57] Costa-Pinto, A. et al., Biomacromolecules 2009.

[58] Puchtler, H. et al., J Histochem Cytochem 1969, 17, 110.

[59] Billington, C. J. Methods Mol Biol 2001, 151, 451.

[60] Alves da Silva, M. L. et al., Acta Biomater 2010, 6, 1149.

[61] Neuman, R. E.; Logan, M. A. Journal of Biological Chemistry 1950, 184, 299.

[62] Gallop, P. M.; Paz, M. A. Physiol Rev 1975, 55, 418.

[63] Samuel, C. S. In Kidney Research: Experimental Protocols; Becker, G. J., Hewitson, T. D., Eds. 2008; Vol. 466, p 223.

[64] Bergman, I.; Loxley, R. Anal Chem 1963, 35, 1961.

[65] Neuman, R. E.; Logan, M. A. Journal of Biological Chemistry 1950, 186, 549. 